Vergleich stehen die Präparate unverändert für weitere Experimente zur Verfügung.

Die Auswertung der Abb. 2 zeigt an dem Beispiel Muskovit-Aluminium, daß eine Genauigkeit von 1\% mit Sicherheit erzielt wird. Um so scharf gezeichnete Bilder zu erhalten, muß der F r a u n$\mathrm{h}$ of e r sche Strahlengang angewandt, außerdem aber müssen in der Dicke völlig einheitliche und unverbogene Glimmerfolien ohne Falten benutzt werden, so daß die Aufspaltung und Schweifbildung der Interferenzflecke bei höheren Ordnungen vermieden wird, wie sie sich bei den in der Literatur veröffentlichten Diagrammen so häufig zeigen.

Wie man leicht überlegt, verhält sich die $b$-Achse von Muskovit zur Gitterkonstanten von Aluminium $a_{\mathrm{Al}}$ wie die für das De by e-Scher rerDiagramm charakteristische Konstante

$$
\frac{I_{\mathrm{hkl}}}{\sqrt{h^{2}+k^{2}+l^{2}}}
$$

zum Abstand $e$ zweier Punkte des Interferenzmusters von Muskovit. Für diesen Abstand $e$ er-, gibt sich aus 56 Messungen $e=3,31_{7} \mathrm{~mm}$. Aus 24 Messungen der Durchmesser des DebyeScherrer-Diagramms erhält man für die charakteristische Konstante:

$$
\text { Const. }=7,36_{8} \mathrm{~mm} \text {. }
$$

Hieraus errechnet sich das Verhältnis der Gitterkonstanten $\mathrm{zu}$

$$
\frac{b_{\text {Musk. }}}{a_{\mathrm{Al}}}=\frac{\text { Const. }}{\mathrm{e}}=2,221 \pm 0,002 .
$$

Hrn. Prof. Dr. W. Kossel danke ich für sein stetes Interesse und für seinen förderlichen Rat.

\title{
Über den Einfluß`von Substituenten auf die Anregung des Benzolringes durch Elektronenstoß in der Glimmentladung
}

\author{
Von Adalbert Woeldike \\ Aus dem Kaiser-Wilhelm-Institut für Physik, Hechingen \\ (Z. Naturforschg. 1, 566-574 [1946]; eingegangen am 14. August 1946)
}

\begin{abstract}
An Benzolabkömmlingen wird das unterschiedliche Verhalten bei Anregung durch Elektronenstoß bzw. durch Licht an Hand der ultravioletten Emissions-Spektren näher untersucht. Dabei ergibt sich, daß das Licht - wie bekannt - ohne Ausnahme den Benzolring im UV anzuregen vermag, während bei Elektronenstoß das nicht der Fall ist. Vielmehr unterliegt hier die Anregbarkeit des Ringes dem Einfluß der Substituenten. Eine systematische Untersuchung läßt erkennen, daß $\mathrm{C}, \mathrm{H}$ und $\mathrm{F}$ sowie $\mathrm{O}$ und $\mathrm{N}$, beide in Einfachbindung, im Substituenten eine Elektronenstoßanregung des Ringes zulassen, während $\mathrm{Cl}, \mathrm{Br}, \mathrm{S}$ sowie $\mathrm{O}$ und $\mathrm{N}$, beide in Doppelbindung, eine solche Anregung unterbinden. An mehrfachsubstituierten Derivaten zeigt sich auch, daß Substituenten, bei denen die Ringanregung ausbleibt, einen stärkeren Einfluß auf den Ring besitzen als die Substituenten, die diese Anregung nicht behindern. Dieses spezifische Verhalten der Moleküle bei Elektronenstoßanregung ermöglicht die optische Erfassung von Energieverlagerungen im Molekül. Es werden kurz die Möglichkeiten für eine Deutung der hier untersuchten Erscheinung betrachtet. Auf Grund der bisherigen Ergebnisse können gewisse Annahmen ausgeschlossen werden. Eine Erlklärung mit Hilfe der Mesomerielehre wird zur Diskussion gestellt.
\end{abstract}

$\mathrm{W}$ ie H. Schüler und der Verf. wiederholt nachweisen konnten ${ }^{1-4}$, gibt es Moleküle, bei denen ein durch Elektronenstoß in der Glimmentladung erzeugtes Emissionsspektrum mit dem energetisch zugehörigen, durch Licht erzeugten Absorptionsspektrum der Lage und dem Aussehen

\footnotetext{
1 Physik. Z. 43, 415 [1942]

2 Physik. Z. 43, 520 [1942].
}

nach nicht identisch ist. Einen Extremfall hierbei stellt die Gruppe von Molekülen ${ }^{\mathbf{1} 5} \mathbf{d a r}$, die in einem bestimmten Energiegebiet überhaupt keine Emission aufzuweisen vermag, obwohl in dem gleichen Bereich eine Absorption auftritt. Diese

\footnotetext{
3 Physik. Z. 44, 335 [1943].

4 Physik. Z. 45, 171 [1944].

5 Physik. Z. 42, 390 [1941].
} 
spezielle Erscheinung wurde vom Verf. experimentell an Benzolderivaten näher untersucht.

Die aromatischen Verbindungen wurden hier deshalb gewählt, weil bei ihnen nicht nur entsprechende Beobachtungen aus früheren Arbeiten ${ }^{1-4}$ mitverwertet werden können, sondern weil auch ihre Spektren experimentell sehr günstig liegen. Denn bekanntlich wird bei den aromatischen Verbindungen durch Anregung des Benzolkerns (Anregung der $\pi$-Elektronen) - wie die Absorption zeigt - u. a. eine ultraviolette Strahlung hervorgerufen, die zwischen 2500 und $3000 \AA^{6}{ }^{6}, 7$ liegt (Lage dieses Spektrums beim unsubstituierten Benzol s. Tab. 1, Nr. 1 sowie ${ }^{5,6}$ ), also in einem Gebiet, das photographisch wesentlich leichter zu erfassen ist als beispielsweise der Absorptionsbereich aliphatischer Körper, der sich um oder unterhalb von $2000 \AA$ nach kürzeren Wellen zu befindet.

Die erste Benzolverbindung nun, bei der das Ausbleiben einer Emission gefunden wurde, war das Chlorbenzol, $\mathrm{C}_{6} \mathrm{H}_{5} \mathrm{Cl}^{5,8}$. Die ultraviolette $\mathrm{Ab}$ sorption, die sich von 2750 bis $2400 \AA$ erstreckt, weist scharfe, wohldefinierte Banden auf ${ }^{6}$ (s. Tab. 1, Nr.2). Die Schwingungsanalyse des Spektrums läßt erkennen, daß Schwingungsdifferenzen in der Größenordnung von $1000 \mathrm{~cm}^{-1}$ auftreten, die von C-C-Schwingungen herrühren, und ermöglicht damit eine èinwandfreie Zuordnung des Spektrums zu der Benzolringanregung. Aus der Schärfe der Absorptionsbanden ergibt sich, daß nicht nur der Grundterm, sondern auch der angeregte Elektronenterm, zu dem der Absorptionsübergang erfolgt, stabil ist, d. h. also: jede der beiden Potentialkurven, die diese Terme darstellen, besitzt eine ausgeprägte Potentialmulde mit einem größeren $\mathrm{Be}-$ reich, in dem sie von eventuell vorhandenen $\mathrm{Ab}$ stoßungskurven nicht durchschnitten wird. Also müßte auch in der Emission - nach dem Franck-Cond on-Prinzip - ein B^ndenspektrum auftreten, zumindest aber, falls die Minima der beiden Potentialkurven gegeneinander etwas verschoben sein sollten, ein Kontinuum. Das Ausbleiben der Emission bei Vorhandensein einer ausgesprochen scharfen Bandenabsorption stellt somit hier und in den folgenden analogen Fällen eine den bisherigen Erfahrungen widersprechende Tatsache dar.

a H. Sponer u. E. Teller, Rev. mod. Physics 13, 76 [1941].

${ }_{7}$ H. S p o n e r, Molekülspektren I (Tab.), Berlin 1935 .
Dieser Befund am Chlorbenzol wird nicht geändert z. B. durch das Einschalten einer $\mathrm{CH}_{2}-$ Gruppe zwischen Chloratom und Benzolring. Denn die UV-Absorption des Benzylchlorids, $\mathrm{C}_{6} \mathrm{H}_{5} \cdot \mathrm{CH}_{2} \cdot \mathrm{Cl}$, weist außer einem Spektrum unterhalb $2200 \AA^{6}$, das hier nicht weiter zu berücksichtigen ist, ein offensichtlich davon unabhängiges Bandenspektrum im Gebiet von 2850 bis $2500 \AA$ auf. Es ähnelt nicht nur im Aussehen dem Chlorbenzol, sondern zwischen intensiven Banden treten auch Schwingungen auf, wie sie beim Chlorbenzol zu finden sind. Das ermöglicht wieder eine eindeutige Zuordnung dieser Absorption zum aromatischen Kern des Benzylchlorids. Auch hier ist somit die UV-Anregung des Ringes durch Licht möglich, während sie beim Elektronenstoß nicht festzustellen war (siehe Tab. 1, Nr.3). Das gleiche läßt sich vom Brombenzol, $\mathrm{C}_{6} \mathrm{H}_{5} \cdot \mathrm{Br}$, sagen. Seine UV-Absorption liegt im Gebiet von 2760 bis $2400 \AA^{6}{ }^{6}$. Eine zugehörige Emission tritt nicht auf $^{5}$ (s. Tab.1, Nr.4). Beim Fluorbenzol ${ }^{9}, \mathrm{C}_{6} \mathrm{H}_{5} \cdot \mathrm{F}$, ergaben Absorptionsaufnahmen eine Absorption, die zwischen ungefähr 2750 und $2400 \AA$ liegt und scharfe Banden aufweist (s. Tab. 1 , Nr. 5 ; s. a. ${ }^{6}$ ). Hier zeigt sich nun, daß das Ausbleiben der ultravioletten Emission durchaus nicht eine charakteristische Erscheinung für halogensubstituierte Benzolderivate ist, wie man zunächst aus den bisherigen Ergebnissen schließen könnte. Denn das Fluorbenzol besitzt eine Emission mit wohldefinierten Banden, die bei ungefähr $2580 \AA$ beginnt. Der kurzwellige Anfang der Hauptbanden ist bei 2650 А. Das Bandenspektrum erstreckt sich bis etwa $2950 \AA$. Hieran schließt sich ein Kontinuum an, das über 3300 A nach Rot zu hinausgeht. Starke Banden der Emission sind mit ebensolchen der Absorption in Überdeckung zu bringen. Das Aussehen beider Spektren ist bezeichnend für Spektren, die ihren Ursprung in der Anregung der $\pi$-Elektronen des Benzolringes haben.

Bei der Untersuchung von Benzolabkömmlingen, die ein oder mehrere Sauerstoffatome im Substituenten enthalten, ergab sich folgendes: Bei der Salicylsäure, $\mathrm{HO} \cdot \mathrm{C}_{6} \mathrm{H}_{4} \cdot \mathrm{COOH}$, die ihre UV-Absorption zwischen 3000 und $2300 \AA^{6}$ hat, ist in

8 Auch W. H. M c Vi cke r u. Mitarb. (J. Chem. Soc. [London] 123, 2147 [1923]) konnten bei ihren Tes l a - Luminescenz-Untersuchungen an Benzolderivaten ein Ausbleiben des Leuchtens bei Chlor- und Nitrobenzol und Benzylchlorid feststellen.

9 Hrn. Dr. H. K o r s c h i n g sei für die Herstellung des Fluorbenzols gedankt. 
diesem Energiebereich durch Elektronenstoßanregung ein Emissionsleuchten, das einer Ringanregung zuzuschreiben wäre, nicht zu bekommen (s. Tab. 1, Nr. 6). Das Vorhandensein einer Hydroxylgruppe (-OH) als zweiter Substituent ist hier ohne Bedeutung, denn auch die Benzoesäure, $\mathrm{C}_{6} \mathrm{H}_{5} \cdot \mathrm{COOH}$, zeigt im UV kein Emissionsleuchten, das von einer Ringanregung stammen könnte. Von den Benzolderivaten, die eine COH-Gruppe als Substituenten haben, ist u. a. Benzaldehyd, $\mathrm{C}_{6} \mathrm{H}_{5} \cdot \mathrm{COH}$, untersucht worden. Hier liegt die Lichtanregung des Benzolringes im Bereich von 2600 bis $2950 \AA^{6}$. Die Berechtigung, diese Absorption den $\pi$-Ringelektronen zuzuordnen, ergibt sich auch hier wieder aus dem Auftreten von Schwingungsdifferenzen, die ihrer Größe nach - z. B. $\omega^{\prime}=1007$ oder $\omega^{\prime}=615 \mathrm{~cm}^{-1}-$ C-C-Schwingungen entsprechen. (Auch die Lage des reinen Elektronensprunges [0-0-Bande bei $\nu=35194 \mathrm{~cm}^{-1}$ ] läßt schon die Zugehörigkeit dieser Absorption zur Ringanregung vermuten.) Eine entsprechende Emission konnte bei diesem Aldehyd nicht festgestellt werden (s. Tab. 1, Nr. 7). $\mathrm{Zu}$ der $\mathrm{COH}-$ Gruppe gehören auch noch die folgenden drei Derivate. Die Absorption des Toluylaldehyds, $\mathrm{CH}_{3} \cdot \mathrm{C}_{6} \mathrm{H}_{4} \cdot \mathrm{COH}$, zeigte neben Banden, die ungefähr zwischen 3500 und 4000 A liegen, ein Bandenspektrum, das etwa bei $2900 \AA$ beginnt und dessen untere Grenze - nach $2000 \AA \mathrm{zu}$ - schwer abzuschätzen ist, da die gesamte UV-Absorption dieses Stoffes äußerst druckabhängig ist und sehr schnell völlig kontinuierlich wird. Das Aussehen der Banden bei 2900 Å ähnelt der entsprechenden Absorption anderer aromatischer Verbindungen und kann der Ringanregung zugeordnet werden. Durch Elektronenstoß wird solch eine ultraviolette Anregung nicht geschaffen (s. Tab. 1, Nr. 8). Genau so verhält sich der Salicylaldehyd, HO . $\mathrm{C}_{6} \mathrm{H}_{4} \cdot \mathrm{COH}$, bei dem man die durch Licht bewirkte Anregung des Benzolringes vermutlich für die Entstehung des scharf gezeichneten Bandenspektrums, das von 2600 bis $2400 \AA$ reicht (s. Tab. 1, Nr.9), verantwortlich machen darf. Denn das zweite Absorptionsspektrum im UV zwischen 3000 und 3500 A stellt ein Kontinuum dar, dem sich am langwelligen Ende schwache und breite Banden anschließen. In Analogie zu den bisherigen Befunden kann es auf Grund seines Aussehens schwerlich mit einer Ringanregung in Zusammenhang gebracht werden. Eher ist dieses Spektrum als eine für aromatische Verbindungen mit einer
COH-Gruppe charakteristischen Absorption anzusehen, da sowohl beim Toluylaldehyd (s. Tạb. 1, Nr. 8) als auch beim Benzaldehyd ${ }^{7}$ (s. Tab. 1, Nr. 7) zwischen 3000 und $4000 \AA$ Banden allerdings ohne Kontinuum zu finden sind. Beim Vanillin, $\mathrm{CH}_{3} \mathrm{O} \cdot \mathrm{C}_{6} \mathrm{H}_{3}(\mathrm{OH}) \cdot \mathrm{COH}$, tritt bei 3000 A eine breite Absorptionsbande auf (s.Tab.1, Nr.10). $\mathrm{Ob}$ diese Bande auch zu der vermuteten charakteristischen Absorption der $\mathrm{COH}$-substituierten Derivate $\mathrm{zu}$ rechnen ist und damit eine weitere Bestätigung für diese Annahme liefert, oder aber ob die Bande dem mehr im Kurzwelligeren liegenden Absorptionsspektrum noch zugehört, ist erst durch ein näheres Studium der Vanillinabsorption klarzustellen. Hier jedoch soll die Absorptionsaufnahme nur bestätigen, daß auch bei dieser Substanz der Benzolring durch Licht angeregt werden kann. Es zeigt sich in der Tat ein Absorptionsspektrum im Gebiet von 2800 bis $2500 \AA$ mit Banden von 2800 bis $2650 \AA$, denen sich ein bis 2500 sich erstreckendes Kontinuum anschließt. In Emission ist eine entsprechende Strahlung nicht zu beobachten (s. Tab. 1, Nr. 10). Die beiden zuletzt genannten Substanzen enthalten außer der COH-Gruppe noch eine Hydroxylgruppe als Substituent. Diese Gruppe ist zwar ohne Einfluß auf das Ausbleiben der ultravioletten Emission des Benzolringes, wie sich am Benzaldehyd und Toluylaldehyd (s. Tab. 1, Nr. 7 u. 8) zeigen läßt. Doch da hinsichtlich der Erfahrungen, die auf dem hier behandelten Gebiet erst noch zu sammeln sind, ein Studium der einzelnen Substituenten angebracht schien, wurden auch Benzolabkömmlinge, die als Substituent nur eine OH-Gruppe besitzen, untersucht. Die einfachste Verbindung dieser Art ist das Phenol, $\mathrm{C}_{6} \mathrm{H}_{5} \cdot \mathrm{OH}$. Die Absorption zeigt neben Banden, die zwischen 2420 und 2080 A liegen und von einer Anregung der Hydroxylgruppe stammen, ein Bandenspektrum im Gebiet von 2800 bis $2480 \AA$ (s. a. ${ }^{6}$ ). Schwingungsdifferenzen, die C-C-Schwingungen entsprechen, und Lage der 0-0-Bande lassen hier wieder auf Zugehörigkeit zur Benzolringanregung schließen. In der Emission tritt nun ein entsprechendes Bandenspektrum von 2750 bis $3050 \AA$, von dort in einem Kontinuum auslaufend, auf ${ }^{2}$ (s. Tab. 1, Nr. 11). In dem Gebiet, das Emission und Absorption gemeinsam haben, d. h. also zwischen 2800 und $2750 \AA$, ist das Absorptions- und Emissionsspektrum völlig identisch. Mit ánderen Worten: Diese Emission kann der Anregung der $\pi$-Elektronen des Benzol- 
ringes einwandfrei zugeschrieben werden. Das p-Kresol, $\mathrm{CH}_{3} \cdot \mathrm{C}_{6} \mathrm{H}_{4} \cdot \mathrm{OH}$, unterscheidet sich vom Phenol nur durch eine Methylgruppe als zweitem Substituenten. Infolgedessen ergibt die Lichtanregung eine der Lage nach dem Phenol sehr ähnliche Absorption, nämlich ein Bandenspektrum zwischen 2850 und $2650 \AA^{7}$. Entsprechend liegt die Emission, die auch beim $p$-Kresol zu erzeugen ist, in derselben Gegend wie beim Phenol: Banden von 2800 bis $3100 \AA$ mit anschließendem Kontinuum $^{2}$ (s. Tab. 1, Nr. 12). Durch eine weitere Substitution einer $\mathrm{CH}_{3}$-Gruppe entsteht z.B. das p-Xylenol, $\left(\mathrm{CH}_{3}\right)_{2} \mathrm{C}_{6} \mathrm{H}_{3} \cdot \mathrm{OH}$. Hier zeigt sich ebenfalls Emission, und zwar eine praktisch kontinuierliche, bei $2800 \AA$ beginnend und über $3500 \AA$ hinaus nach Rot verlaufend. Aber auch das Absorptionsspektrum sieht beim $p$-Xylenol sehr diffus aus. Es erstreckt sich von 2850 bis $2600 \AA$ mit zwei Absorptionsmaxima, eines bei 2800 und das andere zwischen 2700 und $2750 \AA$ (s. Tab. 1, Nr.13). Weiter wurden in dieser Gruppe untersucht: Thymol, $\left(\mathrm{CH}_{3}\right)_{2} \mathrm{CH} \cdot \mathrm{C}_{6} \mathrm{H}_{3}\left(\mathrm{CH}_{3}\right) \cdot \mathrm{OH}$, mit einem Emissionsleuchten von $2800 \AA$ bis über $3400 \AA$ hinaus (s. Tab.1, Nr.14), und Guajakol, $\mathrm{CH}_{2} \mathrm{O} \cdot \mathrm{C}_{6} \mathrm{H}_{4}$. $\mathrm{OH}$. Seine Absorption zeigt im Bereich von 2850 bis $2600 \AA$ Banden; die Emission, die kontinuierlichen Charakter hat, fängt bei $2750 \AA$ an und erstreckt sich nach längeren Wellen zu mit einem Intensitätsmaximum bei $3100 \AA$ (s. Tab. 1, Nr. 15). Nach den bisherigen Erfahrungen besteht die Berechtigung, diese beiden Emissionsspektren der Anregung des Benzolringes zuzuordnen. Das Vorhandensein einer $\mathrm{OCH}_{5}$-Gruppe im Guajakol führte $\mathrm{zu}$ der Untersuchung des Anisols, $\mathrm{C}_{6} \mathrm{H}_{5}$. $\mathrm{OCH}_{3}$, das solch eine Methoxylgruppe als einzigen Substituenten trägt. Hierbei läßt sich klar zeigen, daß die $\mathrm{OCH}_{3}$-Gruppe auf die Anregung des aromatischen Ringes durch Elektronenstoß nicht störend wirkt, denn es tritt eine Emission ąuf, deren Banden zwischen 2700 und 3100 A liegen ${ }^{2}$. Bei Lichtanregung sind Banden, die von diesem Anregungszentrum ausgestrahlt werden, zwischen 2840 und $2600 \AA$ zu finden ${ }^{6}$ (s. Tab 1, Nr.16). An sauerstoffhaltigen Substanzen wurde schließlich noch das Acetophenon, $\mathrm{C}_{6} \mathrm{H}_{5} \cdot \mathrm{CO} \cdot \mathrm{CH}_{3}$, untersucht. Die Absorption weist außer einem Spektrum unterhalb von $2400 \AA$, das dem CO angehört, ein Spektrum im Gebiet von 2830 bis $2600 \AA$ auf ${ }^{6}$. Dieses Spektrum ist auf eine Anregung der $\pi$-Elektronen des Benzolringes zurückzuführen und offensichtlich nur bei Lichtanregung mög- lich, denn in der Glimmentladung war das entsprechende Spektrum nicht zu sehen (s. Tab. 1, Nr. 17).

Zur Reihe der sauerstoffhaltigen Derivate sind auch Nitrobenzol, $\mathrm{C}_{6} \mathrm{H}_{5} \cdot \mathrm{NO}_{2}$, und Nitrotoluol, $\mathrm{CH}_{3} \cdot \mathrm{C}_{6} \mathrm{H}_{4} \cdot \mathrm{NO}_{2}$, zu zählen. Beide zeigen wohl die ultraviolette Absorption, aber keine Emission ${ }^{1}$ (s. Tab. 1, Nr. 18).

Die Untersuchung stickstoffhaltiger Benzolabkömmlinge wurde durch das Verhalten des Nitrobenzols nahegelegt. So zeigt sich im Anilin, $\mathrm{C}_{6} \dot{\mathrm{H}}_{5} \cdot \mathrm{NH}_{2}$, auch für die Elektronenstoßanregung eine Anregungsmöglichkeit des Benzolringes, denn ein Bandenspektrum von 2850 bis $3300 \AA$ mit anschließendem Kontinuum tritt auf $^{2}$. Bei der Lichtanregung waren in diesem Energiegebiet Banden zwischen 3000 und 2600 A zu beobachten ${ }^{\mathbf{1 0}}$ (s. Tab. 1, Nr. 19). Das Vorhandensein von Schwingungsdifferenzen, die C-CSchwingungen entsprechen, ist festzustellen, so daß auch hier wieder in eindeutiger Weise eine Zugehörigkeit dieses Absorptionsspektrums zur Benzolringanregung vorliegt. Aus der Identität der Banden im Überdeckungsbereich von Absorption und Emission ist die gleiche Folgerung für die Zugehörigkeit des Emissionsleuchtens zu ziehen. Beim Diamin, dem p-Phenylendiamin, $\mathrm{C}_{6} \mathrm{H}_{4}\left(\mathrm{NH}_{2}\right)_{2}$, dagegen ist die Zuordnung der Anregungszentren für die ultravioletten Spektren schwieriger. In der Emission erscheint nämlich erst bei $3150 \AA$ nach Rot zu ein Kontinuum mit einem Maximum bei 3600 bis 3700 A. Die Absorption weist ein Spektrum auf, das zwischen 3000 und $3400 \AA$ liegt und aus breiten Banden besteht. Das Spektrum ist sehr druckabhängig und geht schnell vom violetten Ende aus in ein Kontinuum über. Diesem Spektrum ist nach kürzeren Wellen $\mathrm{zu}$ ein zweites, praktisch kontinuierliches vorgelagert, das ebenfalls sehr druckabhängig ist und sich leicht mit dem anderen vereinigt ( $\mathrm{s}$. Tab. 1, Nr. 20). Die Lage des Emissionsspektrums und des langwelligeren Absorptionsspektrums zueinander und das Aussehen beider - der kontinuierliche Charakter der Emission und die Diffusität der Absorption - rechtfertigt die Annahme eines

10 In der Literatur ${ }^{6}$ wird als langwelliges Ende dieses Spektrums $2800 \AA$ angegeben. Dorh kann in starken Emissionsaufnahmen schon bei 2950 A Selbstumkehr festgestellt werden, $d$. h. also, daß zwischen 2800 und $3000 \AA$ noch Absorptionsbanden, die unbedingt zum Anilin gehören, auftreten. Das wird auch durch eigene Absorptionsversuche bestätigt. 


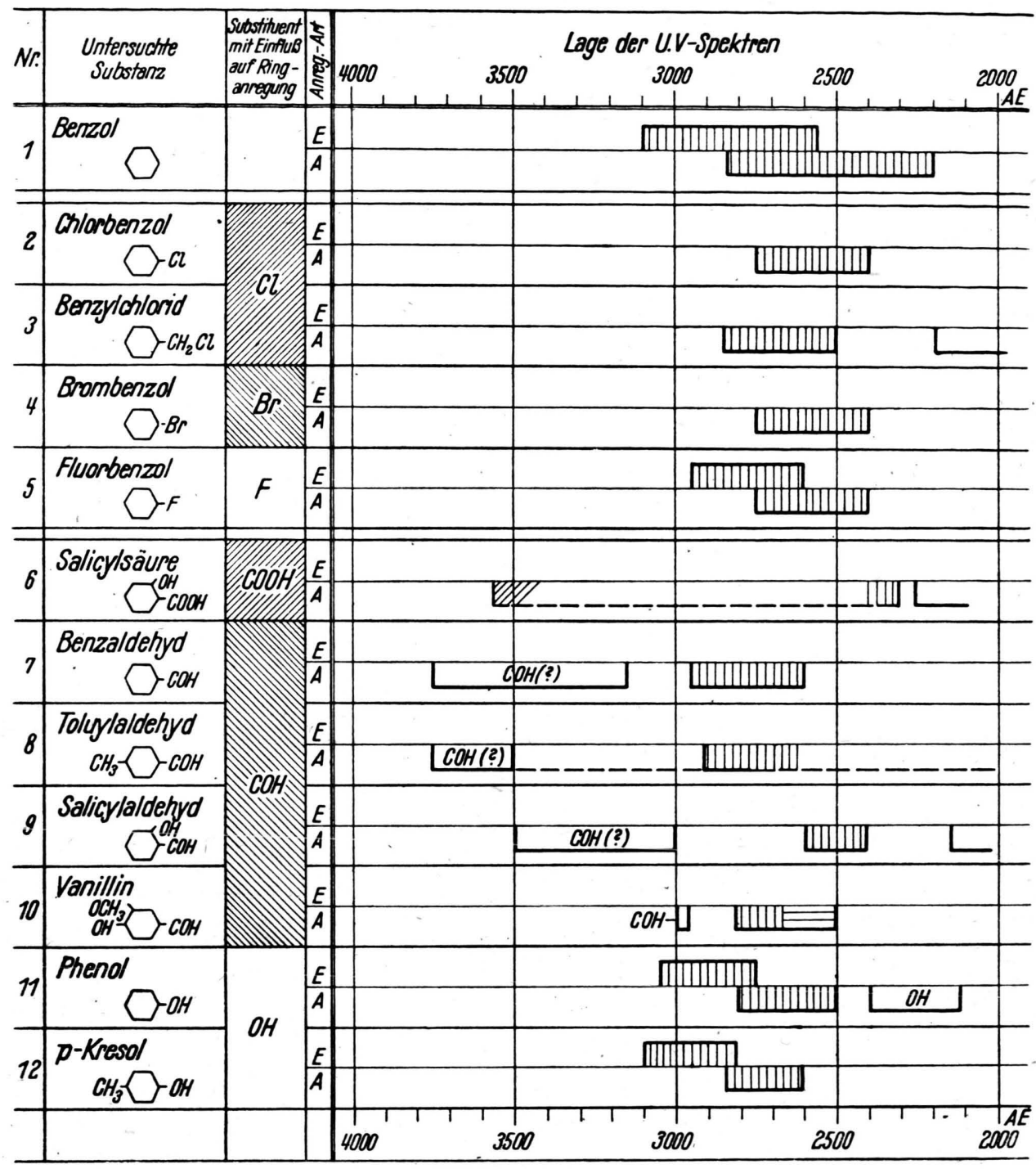

Tab. 1. UV-Spektren von Benzolverbindungen in Emission und Absorption.

$$
\begin{aligned}
& \square \text { bedeutet: Bandenspektrum. } \\
& \square \text { bedeutet: diffuses Spektrum. } \\
& \square \text { bedeutet: kontinuierliches Spektrum. }
\end{aligned}
$$

Zur besseren Übersicht sind in der Substituentenspalte die Substituenten, die den Benzolring für Elektronenstoßanregung sperren, durch Schrägschraffierung hervorgehoben.

Die Daten für die Absorptionsspektren sind, soweit sie nicht eigenen Beobachtungen entstammen, aus ${ }^{6}$ und ${ }^{7}$ entnommen.

gemeinsamen Anregungszentrums für beide Spektren. Dieses Anregungszentrum im Benzolkern zu sehen, könnte zunächst infolge der starken Verschiebung der Spektren nach Rot zu willkürlich erscheinen. Doch schon beim Monoamin reichen die UV-Spektren, die vom Benzolring stammen, weiter in das langwellige Gebiet hinein, als es sonst bei den einfacheren Aromaten üblich ist, und aus Absorptionsuntersuchungen ergibt sich, daß jede weitere Anlagerung eines (gleichartigen) Substituenten eine Verschiebung nach längeren Wellen zu bewirkt, so daß beim Diamin die relativ tiefe energetische Lage verständlich wird. Überhaupt würde die Zuordnung der hier beob- 


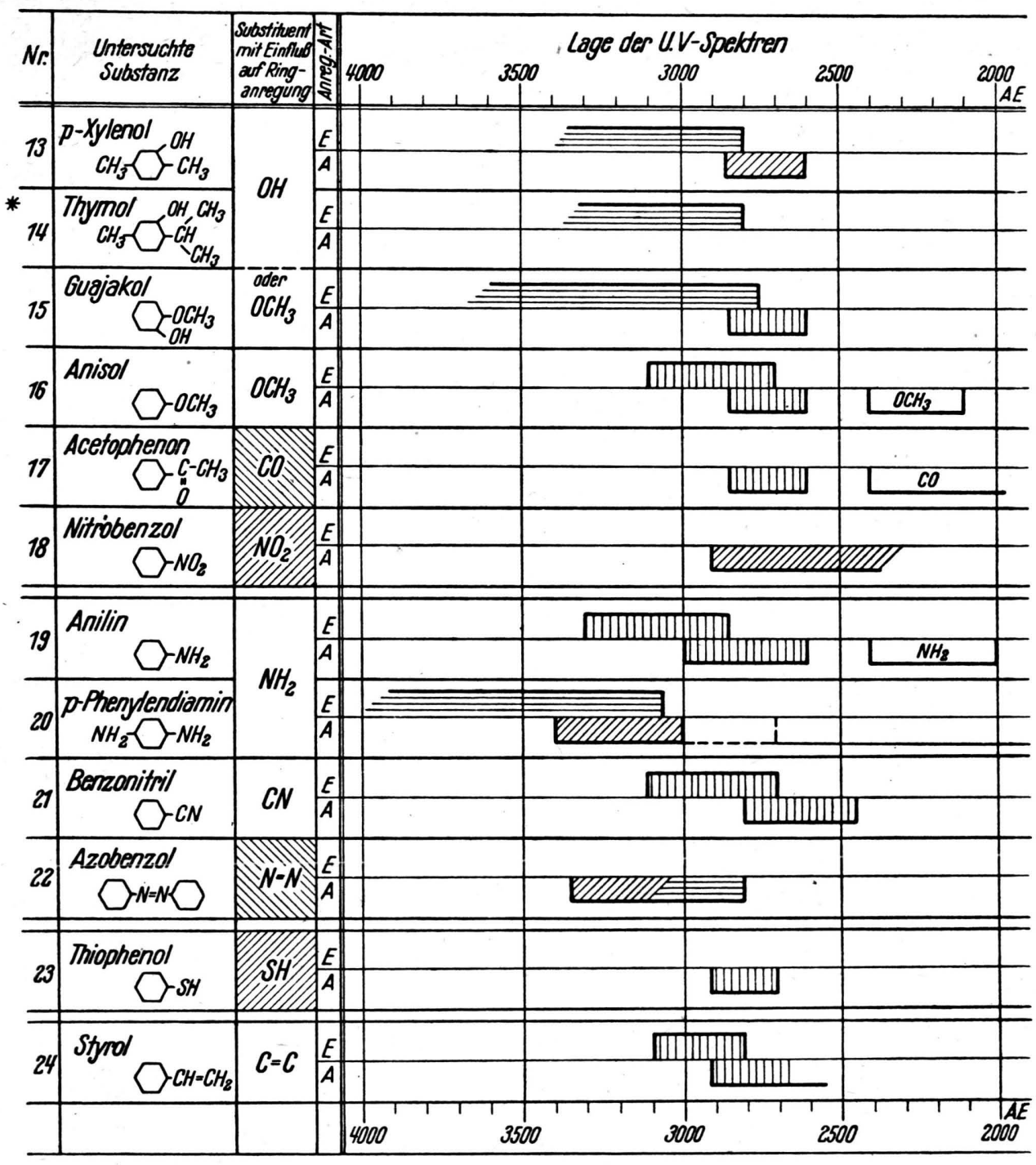

Fortsetzung von Tab. 1.

Zu Nr. 14* Absorption aus technischen Gründen nicht durchgeführt.

achteten Spektren zu einem Anregungszentrum sehr schwierig sein, wenn der Benzolring von vornherein für solche Zuordnung auszuschließen wäre, denn die Aminogruppe, die noch in Frage käme, kann hier nicht in Betracht gezogen werden, da die Anregung der $\mathrm{NH}_{2}$-Gruppe, wie sich z. B. aus der Absorption des Anilins ergibt, zwischen 2400 und 2000 Å liegt. Es besteht also durchaus eine Wahrscheinlichkeit für die Annahme, daß diese Phenylendiaminspektren auf eine Benzolringanregung zurückzuführen sind. Klar ersichtlich ist die Zuordnung wieder beim Benzolnitril, $\mathrm{C}_{6} \mathrm{H}_{5} \cdot \mathrm{CN}$. In der Absorption im Gebiet von 2790 bis $2440 \AA$ lassen sich C-C-Schwin- gungen nachweisen ${ }^{6}$, und mit der Emission, die zwischen 2700 und 3100 A liegt (s. Tab. 1, Nr. 21) ist völlige Identität $\mathrm{zwischen} \mathrm{Absorptions-} \mathrm{und}$ Emissionsbanden festzustellen, d.h. also: beim Benzonitril kann der Benzolring sowohl durch Licht als auch durch Elektronenstoß angeregt werden. Als letzte Substanz dieser Reihe wäre noch das Azobenzol, $\mathrm{C}_{6} \mathrm{H}_{5} \cdot \mathrm{N}_{2} \cdot \mathrm{C}_{6} \mathrm{H}_{5}$, zu erwähnen. Die ultraviolette Absorption ist ziemlich weit nach Rot verschoben, was vielleicht durch die Anwesenheit zweier Benzolringe bedingt ist. Eine UV-Emission trat nicht auf (s. Tab. 1, Nr. 22).

Als drittes Element im Substituenten aromatischer Verbindungen neben Sauerstoff und Stick- 
stoff wurde noch Schwefel untersucht, und zwar in der für vorliegende Betrachtungen einfachsten Form, nämlich das Thiophenol, $\mathrm{C}_{6} \mathrm{H}_{5} \cdot \mathrm{SH}$. Die Lichtanregung des Benzolringes scheint möglich, dafür spricht das Auftreten eines Absorptionsspektrums zwischen 2700 und $2900 \AA$. Die entsprechende Emission blieb aus (s. Tab. 1, Nr. 23).

Zum Schluß ist noch das Styrol, $\mathrm{C}_{6} \mathrm{H}_{5} \cdot \mathrm{CH}: \mathrm{CH}_{2}$, zu nennen. In der Emission konnte ein Spektrum beobachtet werden, dessen Banden zwischen 2800 und $3100 \AA$ Å lagen; das anschließende Kontinuum erstreckte sich bis über $3600 \AA$ hinaus. Absorptionsaufnahmen direkt wurden von dieser Verbindung nicht gemacht. Doch zeigt sich bei starken Emissionsaufnahmen Selbstumkehr im Gebiet von $2880 \AA$, so daß man in dieser Gegend das rote Ende der zugehörigen UV-Absorption anzunehmen hat (s. Tab. 1, Nr. 24). Die Spektren sind der Lage und dem Aussehen nach so typisch für die UV-Spektren der Benzolderivate, daß man sie ohne weiteres der Anregung des Benzolringes zuschreiben kann.

Diese Ergebnisse sollen nun vom Standpunkt des Substituenteneinflusses auf die Benzolkernanregung aus kurz zusammengefaßt werden. Dazu sei auch auf Tab. 1 hingewiesen, in der zur besseren Übersicht in einer besonderen Spalte immer derjenige Substituent eingetragen ist, dem der Haupteinfluß auf die Kernanregbarkeit zugeschrieben werden muß. Danach ergibt sich also, daß bei der Elektronenstoßanregung - das Verhalten bei Lichtanregung soll hier zunächst unberücksichtigt bleiben - Chlor und Brom eine Nichtanregung des benzoiden Kernes bewirken, während Fluor eine Anregung zuläßt, sich also genau so verhält wie Wasserstoff und Kohlenstoff im Substituenten. Beim Sauerstoffatom zeigt sich, daß nicht nur die Anwesenheit eines bestimmten Atoms im Substituenten entscheidend ist. sondern auch die Art, wie das Atom dort gebunden ist, ob also eine Einfachbindung oder eine Doppelbindung am Atom vorliegt. Benzolderivate, bei denen am Sauerstoffatom nur Einfachbindung vorhanden ist, etwa die OH-Gruppe, weisen alle eine Emission auf (Phenol, Anisol). Dagegen ist bei O-Atomen mit Doppelbindung, wie z. B. in der COH-Gruppe und COOH-Gruppe, eine UV-Emission nicht zu beobachten. Weiter ist zu erkennen,

11 Tautomerie u. Mesomerie, Stuttgart 1938.

12 Theoretische Grundlagen d. org. Chemie II, Leipzig 1941. daß Substituenten mit doppeltgebundenem O-Atom einen größeren Einfluß auf die Ringanregung haben als die Substituenten, die eine Ringanregung zulassen. Denn Aromate mit mehreren Substituenten zeigen, sobald auch nur einer von den Substituenten ein Sauerstoffatom mit Doppelbindung enthält, keine ultraviolette Anregung mehr. Auch im Substituenten selbst, wenn er mehr als ein O-Atom besitzt, hat das doppeltgebundene O-Atom die gleiche entscheidende Bedeutung. Ein Beispiel dafür ist die $\mathrm{COOH}-G r u p p e$ und die Nitrogruppe, bei der ja nach den Anschauungen der modernen Elektronentheorie eines der O-Atome immer dem Stickstoff in Doppelbindung anliegt (z. B. $\left.\stackrel{\mathrm{N}}{\mathrm{J}}\right|^{\mathrm{N}}=\underline{\mathrm{O}}$; s. B. E is te r t ${ }^{11}$ oder W. H ü c k e ${ }^{12}$ ). Vielleicht kann sogar auch umgekehrt das hier aufgefundene Verhalten der Nitrogruppe als eine weitere Bestätigung für die Zulässigkeit dieser elektronentheoretischen Annahme angesehen werden. Weiter ist aus den Befunden an der $\mathrm{NO}_{2}$-Gruppe noch zu ersehen, daß der bestimmende Einfluß des doppeltgebundenen Sauerstoffatoms auch dem Stickstoffatom gegenüber bestehen bleibt, denn Stickstoff im sauerstofffreien Substituenten wirkt durchaus nicht störend auf die Anregung des Benzolringes (Anilin), auch dann nicht, wenn es in Dreifachbindung vorliegt wie in der CN-Gruppe (Benzonitril). Nur im Falle einer Doppelbindung zwischen zwei N-Atomen scheint Stickstoff die UV-Anregung des Benzolringes zu unterbinden (Azobenzol) ${ }^{13}$. Ähnlich wie Chlor und Brom muß sich das Schwefelatom verhalten, denn auch bei ihm sieht es so aus, als ob die Ringanregung gesperrt wird.

Diese Zusammenstellung zeigt darüber hinaus, daß das Studium der Emission mehr zu bringen vermag als nur eine Ergänzung der Absorption. Denn während bei der Anregung der Benzolderivate durch Licht ohne Ausnahme - wie ja eingangs auch erwähnt - ultraviolette Spektren auftreten, die sich durch Lage und Schwingungsgröße voneinander unterscheiden, bewirkt die Elektronenstoßanregung zusätzlich ein spezielles Verhalten der Moleküle und trägt damit ein neues

13 Eine während der Niederschrift des Manuskripts durchgeführte Untersuchung am Diphenylamin, $\mathrm{C}_{6} \mathrm{H}_{5}$ - $\mathrm{NH} \cdot \mathrm{C}_{6} \mathrm{H}_{5}$, ergab, daß diese Suhstanz im UV eine kontinuierliche Emission von $3000 \AA$ ah nach Rot zu besitzt, was als eine weitere Bestätigung des hier aufgezeigten Verhaltens der N-Atome angesehen werden kann. 
Moment in das Studium organischer Moleküle hinein.

Es dürfte nur schwer möglich sein, für die hier untersuchte Erscheinung schon jetzt eine völlige Klärung zu erlangen, allein schon auf Grund der Tatsache, daß die Strahlung, die von höheren Elektronenübergängen herrührt - also z. B. die Emission im Sch u m an - Gebiet - , aus tęchnischen Gründen noch nicht untersucht werden konnte, und somit wichtige Aussagen zunächst noch fehlen. Jedoch ist es naheliegend, die bisher gesammelten Erfahrungen für eine Diskussion verschiedener Deutungsmöglichkeiten heranzuziehen. So wäre eine triviale Deutung für das Ausbleiben der Emission die Annahme einer so großen Instabilität der angeregten Zustände, daß auss diesen Anregungszuständen heraus eine Emission nicht stattfinden kann. Dagegen sprechen nun z. B. die Schärfe der Chlorbenzolabsorption - wie eingangs näher auseinandergesetzt wurde - und andere in diesem Zusammenhang beobachtete Absorptionsspektren. Weiter wurde versucht, das Nichterscheinen der Emission auf einen verbotenen Übergang zurückzuführen. Diese Annahme ist aher einmal mit der Tatsache, daß das Licht stets den Benzolring anzuregen vermag, gleichviel welcher Substituent anhängt, nicht ohne weiteres in Einklang zu bringen; zum anderen läßt sich z. B. am Benzol der geringe Einfluß eines verbotenen Überganges auf die Kernanregung klar erkennen. Denn die hier beobachteten UV-Spektren

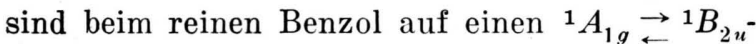
Übergang zurückzuführen, der an und für sich verboten ist, durch eine antisymmetrische Schwingung aber wieder erlaubt gemacht wird ${ }^{14}$. Wenn also schon beim reinen Benzol eine solche antisymmetrische Schwingung vorhanden ist, wieviel mehr müssen derartige Schwingungen dann im einoder mehrfach-substituierten Benzol auftreten und damit verbotenen Übergängen jede praktische Bedeutung nehmen. - Man könnte zunächst auch bei diesem Nichterscheinen der Emission, wie etwa beim Chlorbenzol $\left(\mathrm{C}_{6} \mathrm{H}_{5} \mathrm{Cl}\right)$ an eine ,sterische Hinderung" denken. Doch zeigt der Befund z. B. am Benzylchlorid $\left(\mathrm{C}_{6} \mathrm{H}_{5} \cdot \mathrm{CH}_{2} \cdot \mathrm{Cl}\right)$, bei dem ja die Emission auch ausbleibt, daß eine solche Hinderung keinen prinzipiellen Einfluß auf die Sperrung der Kernanregung haben kann. Ebenso ist es nicht möglich, die Gruppe von Atomen, die eine Ringanregung zuläßt, und die Gruppe, bei

14 A. L. S k l a r, J. chem. Physics 5, 669 [1937] der eine Ringanregung ausbleibt, mit irgend welchen chemischen Reihen, wie sie aus der anorganischen Chemie bekannt sind, und in denen die Elemente auf Grund ihrer chemischen Verwandtschaft zusammengefaßt sind, $\mathrm{zu}$ vereinigen. Denn was z. B. die Halogene anbetrifft, so verhalten sich Chlor und Brom im vorliegenden Fall ja anders als Fluor; genau so ist es auch mit der Reihe Sauerstoff, Schwefel usw. bestellt. O und S könnte man nach ihrem hier aufgefundenen Verhalten schwerlich in eine gleiche Gruppe bringen.

Jedoch eine Tatsache scheint für eine Deutung vorläufig beachtenswert zu sein. Sieht man nämlich zunächst von den chlor-, brom- und schwefelsubstituierten Benzolderivaten ab, so findet man, daß im Substituenten dieselben Atome ( $\mathrm{O}$ und $\mathrm{N})$, die bei Einfachbindung die Ringanregung durch Elektronenstoß zulassen, diese Ringanregung, sobald ihnen eine Doppelbindung anliegt, plötzlich sperren; oder anders ausgedrückt: bei Derivaten mit N- und O-haltigen Substituenten tritt keine Emission auf, wenn sich vom Substituenten aus in den Benzolkern hinein eine sogenannte konjugierte Doppelbindung erstreckt. Das Vorhandensein solch einer konjugierten Doppelbindung allein kann jedoch nicht das Ausbleiben der Emission erklären, wie sich am Beispiel des Styrols, $\mathrm{C}_{6} \mathrm{H}_{5} \cdot \mathrm{CH}: \mathrm{CH}_{2}$, mit seiner ausgeprägten ultravioletten Emission zeigen läßt. Der bindungsabhängige Einfluß der Atome im Substituenten auf die Ringanregung bleibt aber dennoch als eine auffallende Erscheinung bestehen. Es scheint daher, d. h. immer nur in Anbetracht der bisherigen Befunde, nicht abwegig, einmal zur Diskussion $\mathrm{zu}$ stellen, ob es möglich ist, dieses Phänomen der Benzolkernanregbarkeit mittels Vorstellungen aus der modernen Elektronentheorie, insbesondere mit Hilfe der Mesomerielehre zu erklären.

So könnte man z. B. annehmen, daß für die Elektromenstoßanregung nur eine ganz bestimmte Art von Grenzanordnungen wesentlich sind, etwa die polaren Grenzstrukturen, die in zwei Gruppen zu zerlegen wären, von denen die eine die Ringanregung zuläßt und die andere die Ringanregung sperrt. Für die Lichtanregung müßte dann wegen des einheitlichen Auftretens der Absorptionsspektren andere Grenzformeln angenommen werden (z. B. die von Schwarzenbach u. Mitarbeitern ${ }^{15}$ als Normalformeln bezeichneten Struk-

15 G. S chw arzenbach, K. Lutz u. E. Fel d e r, Helv. chim. Acta 27, 576 [1944]. 
turen), was an und für sich auch mit den Anschauungen der Mesomerielehre übereinstimmen würde. Denn es wird allgemein als gesichert angesehen, daß Grenzanordnungen von der Art der polaren Strukturen für die Lichtanregung unbedeutend sind, zumal wenn nur die niedrigen Anregungszustände des Moleküls für die Absorption in Betracht zu ziehen sind (s. z. B. ${ }^{15}$ und E. M ülle r 16, Kapitel: Konstitution und Farbe). Weiter müßte noch gefordert werden, daß die Substituenten, bei denen eine Anregung des Ringes möglich ist, wie $\mathrm{OH}, \mathrm{OCH}_{3}, \mathrm{NH}_{2}$ usw., einander ähnliche Ladungsverteilungen im Ring schaffen können, ebenso wie die andere Gruppe von Substituenten, die eine Kernanregung unterbinden, also $\mathrm{NO}_{2}, \mathrm{COH},-\mathrm{N}=\mathrm{N}-$ usw., Ladungsverteilungen, die sich entsprechen, erzeugen muß. Nun ist es in der Mesomerielehre möglich, die Atomgruppen und Substituenten in bezug auf

18 Neuere Anschauungen der org. Chemie, Berlin 1940.

17 Auch in der Farbstofflehre findet man eine entsprechende Aufteilung - von einigen Ausnahmen wie z. B. der amphoteren Gruppe $-\mathrm{C}=\mathrm{C}-$ abgesehen Die beiden Gruppen werden hier bekanntlich als Auxochrome und Antiauxochrome bezeichnet (siehe Wi z i ng e r, Organische Farbstoffe, Bonn 1933). Dazu näher Stellung zu nehmen, dürfte jedoch über den Rahmen des hier Vorgesehenen hinausgehen.
Schaffung von Ladungsverteilungen im Molekül in zwei Gruppen einzuteilen, und zwar je nachdem, ob der Substituent bzw. die Atomgruppe ein $\pi$-Elektronenpaar dem übrigen Molekülteil zurVerfügung stellt oder aber herauszieht $[(+) E$ - und (一) $E$-Effekt $\left.{ }^{10,16}\right]$. Und diese Einteilung ist praktisch die gleiche wie die, die auf Grund der vorliegenden Untersuchungen an den Substituenten vorgenommen werden konnte ${ }^{17}$. Was dagegen mit dieser Heranziehung von polaren Grenzstrukturen nach den bisherigen Erfahrungen u. a. nicht erklärt werden könnte, ist das hier beschriebene Verhalten von Chlor und Brom bzw. Schwefel als Substituenten.

Unabhängig von allen solchen oder ähnlichen Erwägungen aber ist die Tatsache, daß der hier beobachtete Einfluß eines einzelnen Substituenten auf ein oft mehrfach substituiertes aromatisches System immer nur als eine Energieverlagerung im Molekül verstanden werden kann, so daß die vorliegende Untersuchung im wesentlichen einen weiteren Beitrag zur experimentellen Erfassung von Energieverlagerungen im Molekül darstellt.

Hrn. Prof. Dr. H. Sch ül er möchte ich herzlich danken für das große Interesse an dieser Arbeit, für die großzügige Bereitstellung apparativer Hilfsmittel sowie für die Möglichkeit einer langjährigen Mịtarbeit an seinen molekülphysikalischen Untersuchungen.

\title{
Notiz zur Thermochemie des Bors
}

\author{
Von W. A. Roth \\ (Z. Naturforschg. 1, 574-576 [1946]; aus Grenzach, Baden, eingegangen am 17. Juni 1946)
}

\begin{abstract}
$\mathrm{Zwei}$ moderne Arbeiten über die Bildungswärmen von glasigem $\mathrm{B}_{2} \mathrm{O}_{3}$ werden kritisch durchgerechnet; mit Hilfe der verbesserten Werte werden die Bildungswärmen der bisher thermisch untersuchten Borverbindungen neu berechnet und mit den Werten für analoge Verbindungen der Nachbarelemente verglichen.
\end{abstract}

$\mathrm{V}_{\mathrm{is}}$ on den Elementen der ersten Horizontalreihe ist das Bor als der nichtmetallische Vorläufer der typisch metallischen, dreiwertigen Elemente wohl am merkwürdigsten, schon durch die Existenz von Verbindungen wie $\left(\mathrm{B}_{2} \mathrm{H}_{6}\right)$ und der starken Säure $\mathrm{HBF}_{4}$. Thermisch ist das Bor wenig untersucht.

Mit elementarem Bor sind bisher erst drei Versuchsreihen ausgeführt worden: Troost und 1 Ann. chim. phys. (5) 9, 77 [1876].
Ha u te f e u ille ${ }^{1}$ chlorierten 1876 (wahrscheinlich unreines) Bor, $\mathrm{R}$ o t $\mathrm{h}$ und Mitarbeiter ${ }^{2}$ oxydierten 1937 reines Bor bei etwa $20^{\circ} \mathrm{C}$ in der calorimetrischen Bombe mit Paraffinöl als Hilfssubstanz

2 W. A. Roth u. Erika Böger, Ber. dtsch. chem. Ges. 70, 48, und (mit A. B e r tram) 971 [1937], wo die Lösungs- und Hydratationswärmen richtiggestellt werden; die Resultate sind fast identisch mit den von M.v.St a c kelberg, F. Qu a tra m u. J.Dressel (Z. Elektrochem. angew. physik. Chem. 43, 14 [1937]) gefundenen. 